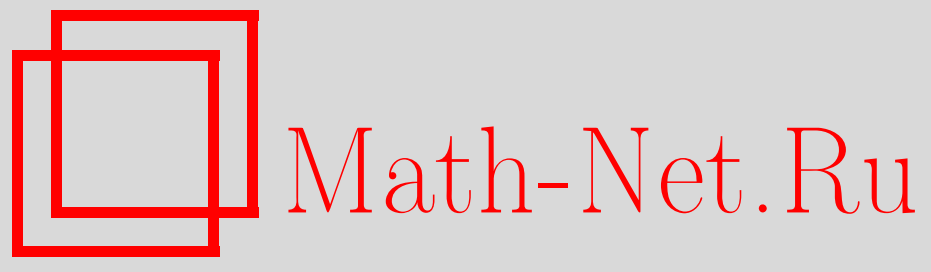

М. С. Еряшкин, С. М. Скрябин, Наибольшая подалгебра Хопфа в биалгебре, Матем. заметки, 2009, том 86, выпуск 6, 942-946

DOI: https://doi.org/10.4213/mzm8528

Использование Общероссийского математического портала Math-Net.Ru подразумевает, что вы прочитали и согласны с пользовательским соглашением http://www . mathnet.ru/rus/agreement

Параметры загрузки:

IP : 54.92.164.108

26 апреля 2023 г., 13:33:03

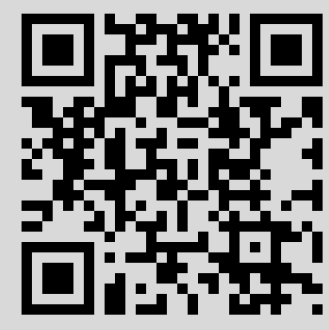




\section{Наибольшая подалгебра Хопфа в биалгебре}

\section{М. С. Еряшкин, С. М. Скрябин}

Как известно, алгебры Хопфа выделяются среди биалгебр наличием антипода - обратного к тождественному отображению относительно операции конволюции. Для двух подалгебр Хопфа $H_{1}, H_{2}$ биалгебры $B$ не ясно, всегда ли существует подалгебра Хопфа, содержащая $H_{1}$ и $H_{2}$ одновременно. Основной результат настоящей работы заключается в том, что ответ на этот вопрос положителен, если $B$ слабо конечна, и в этом случае $B$ имеет наибольшую подалгебру Хопфа $\mathscr{H}(B)$. Для произвольной алгебры Хопфа $H$ образ любого гомоморфизма биалгебр $H \rightarrow B$ содержится в $\mathscr{H}(B)$.

Слабо конечными являются многие классы колец (см., например, [1] или [2]). В частности, сформулированный выше результат применим к любой нётеровой слева или справа биалгебре, а также к любой биалгебре, удовлетворяющей полиномиальному тождеству. По крайней мере, утверждение о гомоморфизмах $H \rightarrow B$ перестает быть верным без предположения о слабой конечности.

Следствие 2.4 показывает, что биидеал $J$ алгебры Хопфа $H$ с необходимостью является идеалом Хопфа (т.е. факторбиалгебра $H / J$ является алгеброй Хопфа) при условии, что $H / J$ слабо конечна. Это усиливает результат работы [3].

1. Наибольшая обратимая подкоалгебра. Все рассматриваемые алгебры и коалгебры определены над полем $k$. Алгебры предполагаются ассоциативными с единицей, коалгебры - коассоциативными с коединицей. Через $\Delta: C \rightarrow C \otimes C$ будем обозначать коумножение, а через $\varepsilon: C \rightarrow k$ - коединицу коалгебры $C$. Если $A$ - алгебра, то векторное пространство $\operatorname{Hom}(C, A)$ всех линейных отображений $C \rightarrow A$ наделено структурой алгебры посредством конволюции $*$, задаваемой по формуле

$$
\varphi * \psi=M \circ(\varphi \otimes \psi) \circ \Delta, \quad \varphi, \psi \in \operatorname{Hom}(C, A),
$$

где $M: A \otimes A \rightarrow A-$ умножение в $A$ (см. [4], [5]). Единичным элементом этой алгебры служит отображение $u \circ \varepsilon$, где $u: k \rightarrow A$ определено сопоставлением $\alpha \mapsto \alpha 1_{A}$. В случае, когда $\operatorname{dim} C<\infty$, имеется канонический изоморфизм алгебр $\operatorname{Hom}(C, A) \cong C^{*} \otimes A$. Если $g: D \rightarrow C$ - морфизм коалгебр, а $f: A \rightarrow B$ - морфизм алгебр, то отображение

$$
\operatorname{Hom}(g, f): \operatorname{Hom}(C, A) \rightarrow \operatorname{Hom}(D, B), \quad \varphi \mapsto f \circ \varphi \circ g,
$$

является морфизмом алгебр. В частности, для обратимого элемента $\varphi$ алгебры $\operatorname{Hom}(C, A)$ с обратным $\varphi^{-1}$ имеем $f \circ \varphi^{-1} \circ g=(f \circ \varphi \circ g)^{-1}$ в $\operatorname{Hom}(D, B)$.

Пусть далее $B$ - некоторая биалгебра. Если $H$ - подалгебра Хопфа в $B$, то ее антипод $s_{H}$, рассматриваемый как линейное отображение $H \rightarrow B$, является обратным к отображению включения $H \rightarrow B$ в алгебре $\operatorname{Hom}(H, B)$. Это мотивирует следующее

ОПредЕЛЕние 1. Назовем подкоалгебру $C \subseteq B$ слабо обратимой в $B$, если отображение включения $i: C \rightarrow B$ обратимо в $\operatorname{Hom}(C, B)$. Обозначим $s_{C}=i^{-1}$. Назовем $C$ обратимой в $B$, если, кроме того, $\operatorname{Im} s_{C} \subseteq C$.

Лемма 1.1. Линейное отображение $\varphi: C \rightarrow A$ обратимо относительно $*$ в каждом из следующих случаев:

Работа выполнена при поддержке Российского фонда фундаментальных исследований (грант № 07-01-00222).

(C) М. С. Еряшкин, С. М. Скрявин, 2009 
(а) $C=\underline{\lim } C_{\alpha}$ есть прямой предел индуктивной системы коалгебр $\left(C_{\alpha}, \mu_{\alpha \beta}\right), u$ $\varphi_{\alpha}=\overrightarrow{\varphi \circ} \mu_{\alpha}$ обратимо в $\operatorname{Hom}\left(C_{\alpha}, A\right)$ для каждого индекса $\alpha$, где $\mu_{\alpha}: C_{\alpha} \rightarrow C-$ каноническое отображение;

(b) $C=\sum C_{\alpha}$ есть сумма некоторого семейства подкоалгебр, причем $\left.\varphi\right|_{C_{\alpha}}$ обратимо в $\operatorname{Hom}\left(C_{\alpha}, A\right)$ для каждого $\alpha$.

Доказательство. Пусть $\psi_{\alpha}=\varphi_{\alpha}^{-1}$ в $\operatorname{Hom}\left(C_{\alpha}, A\right)$. В случае (а) $\varphi_{\alpha}=\varphi_{\beta} \circ \mu_{\alpha \beta}$ для каждой пары индексов $\alpha \leqslant \beta$; так как $\mu_{\alpha \beta}: C_{\alpha} \rightarrow C_{\beta}$ - морфизм коалгебр, то $\psi_{\alpha}=$ $\psi_{\beta} \circ \mu_{\alpha \beta}$. Следовательно, существует единственное линейное отображение $\psi: C \rightarrow A$ такое, что $\psi_{\alpha}=\psi \circ \mu_{\alpha}$ для всех $\alpha$. Так как $\mu_{\alpha}-$ морфизм коалгебр, то $(\varphi * \psi) \circ \mu_{\alpha}=$ $\varphi_{\alpha} * \psi_{\alpha}$ есть единица алгебры $\operatorname{Hom}\left(C_{\alpha}, A\right)$, т.е. $u \circ \varepsilon \circ \mu_{\alpha}$. Отсюда $\varphi * \psi=u \circ \varepsilon$, и аналогично $\psi * \varphi=u \circ \varepsilon$. Значит, $\psi=\varphi^{-1}$ в $\operatorname{Hom}(C, A)$.

В случае (b) рассмотрим множество $\mathscr{G}$ всех подкоалгебр $D \subseteq C$ таких, что $\left.\varphi\right|_{D}$ обратимо в $\operatorname{Hom}(D, A)$. Проверим, что $D_{1}+D_{2} \in \mathscr{G}$ для любых $D_{1}, D_{2} \in \mathscr{G}$. Обозначая $D=D_{1}+D_{2}$ и $\psi_{i}=\left(\varphi \mid D_{i}\right)^{-1}$, имеем

$$
\left.\psi_{i}\right|_{D_{1} \cap D_{2}}=\left(\left.\varphi\right|_{D_{1} \cap D_{2}}\right)^{-1} \quad \text { для } \quad i=1,2 .
$$

Поэтому $\psi_{1}$ совпадает с $\psi_{2}$ на $D_{1} \cap D_{2}$, и существует линейное отображение $\psi: D \rightarrow A$ такое, что $\psi_{i}=\left.\psi\right|_{D_{i}}$ для $i=1,2$. Отображения $\left.\varphi\right|_{D} * \psi$ и $\left.\psi * \varphi\right|_{D}$ совпадают с $u$ о $\varepsilon$ на $D_{1}$ и на $D_{2}$, откуда $\psi=\left(\left.\varphi\right|_{D}\right)^{-1}$. Таким образом, множество $\mathscr{G}$, упорядоченное по включению, есть индуктивная система коалгебр. Так как $C_{\alpha} \in \mathscr{G}$ для всех $\alpha$, то

$$
C=\sum_{D \in \mathscr{G}} D=\underline{\lim _{\longrightarrow}} \mathscr{G},
$$

и можно воспользоваться (а).

Следствие 1.2. Сумма $C=\sum C_{\alpha}$ любого семейства (слабо) обратимых подкоалгебр в $B$ является (слабо) обратимой подкоалгеброй.

ДокАзАТЕЛЬСтво. Применяя лемму 1.1 к отображению включения $C \rightarrow B$, получаем, что подкоалгебра $C$ слабо обратима в $B$. Так как ограничение $s_{C}$ на $C_{\alpha}$ совпадает с $s_{C_{\alpha}}$, то $\operatorname{Im} s_{C}=\sum \operatorname{Im} s_{C_{\alpha}}$. Если $\operatorname{Im} s_{C_{\alpha}} \subseteq C_{\alpha}$ для всех $\alpha$, то $\operatorname{Im} s_{C} \subseteq C$.

Обозначим через $B^{\text {ор }}$ биалгебру, полученную из $B$ изменением умножения на противоположное $\left(a \cdot_{B^{\text {op }}} b=b \cdot{ }_{B} a\right.$ для $\left.a, b \in B\right)$.

ПредложениЕ 1.3. Любая биалгебра $B$ содержит наибольшую обратимую в $B$ подкоалгебру. Кроме того, В содержит наибольшую подкоалгебру, обратимую как в $B$, mak $u$ в $B^{\text {op }}$.

ДокАзАТЕЛЬСтво. Применим следствие 1.2 к множеству всех подкоалгебр, обратимых в $B$ в одном случае и обратимых одновременно в $B$ и в $B^{\text {op }}$ в другом случае.

Без дополнительных теоретико-кольцевых предположений не удается доказать, что подкоалгебры, существование которых установлено в предложении 1.3, замкнуты относительно умножения в $B$.

2. Подалгебры Хопфа в слабо конечных биалгебрах. Кольцо $R$ называется слабо (или стабильно) конечным, если для любого целого $n>0$ и любых $(n \times n)$-матриц $X, Y \in \operatorname{Mat}_{n}(R)$ равенство $X Y=1$ влечет равенство $Y X=1$. Напомним, что $R$-модуль

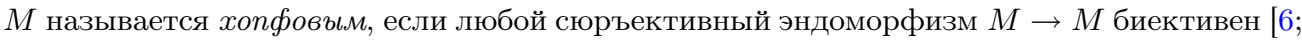
упражнение 1.12]. Таким образом, $R$ слабо конечно тогда и только тогда, когда свойство Хопфа выполнено для всех конечно порожденных свободных правых $R$-модулей, причем данное условие на правые $R$-модули равносильно аналогичному условию на левые 
$R$-модули. В [7] найдена характеризация колец, для которых каждый конечно порожденный правый модуль хопфов. Этот класс колец содержит все нётеровы справа кольца, а также все кольца, являющиеся справа конечно порожденным модулем над коммутативным подкольцом. В частности, такие кольца слабо конечны. Слабая конечность колец, удовлетворяющих полиномиальному тождеству, доказана в [1; с. 599, следствие 1].

Лемма 2.1. Пусть $R$ - слабо конечная алгебра, $A$ - конечномерная алгебра, $A^{\prime}$ подалгебра в $A$. Если элемент $x \in A^{\prime} \otimes R$ обратим в алгебре $A \otimes R$, mо $x^{-1} \in A^{\prime} \otimes R$.

ДоказАтельство. Отождествим $R$ и $N=A^{\prime} \otimes R$ с подалгебрами тензорного произведения алгебр $M=A \otimes R$. Отображение $q: M \rightarrow M, m \mapsto x m$, имеет обратное отображение $m \mapsto x^{-1} m$. Значит, $q$ биективно. Рассмотрим $M$ как $R$-модуль относительно правых умножений. Тогда $q \in \operatorname{End}_{R} M$. Так как $q(N)=x N \subseteq N$, то $q$ индуцирует сюръективный $R$-линейный эндоморфизм $\widetilde{q}: M / N \rightarrow M / N$. Поскольку $R$-модуль $M / N \cong A / A^{\prime} \otimes R$ свободен и конечно порожден, то $\widetilde{q}$ биективен в силу условия слабой конечности. Следовательно, $q^{-1}(N)=N$. Из включения $1 \in N$ выводим $x^{-1}=q^{-1}(1) \in N$.

ЗАмечание. Если алгебра $R$ нётерова слева, то лемма 2.1 может быть доказана следующим образом. Так как алгебра $N=A^{\prime} \otimes R$ нётерова слева, а $M=A \otimes R$ - нётеров $N$-модуль относительно левых умножений, то $x^{-n} \in \sum_{i=0}^{n-1} N x^{-i}$ для достаточно большого $n>0$. Умножая справа на $x^{n-1}$, находим $x^{-1} \in N$. Похожее рассуждение применимо в случае, когда $R$ - конечно порожденный модуль над коммутативным подкольцом.

Лемма 2.2. Пусть $B$ - слабо конечная биалгебра, $C$ - коалгебра, $\varphi: C \rightarrow B$ - морфизм коалгебр. Если $\varphi$ - обратимый элемент алгебры $\operatorname{Hom}(C, B)$, то подкоалгебра $\varphi(C)$ слабо обратима в $B$.

Доказательство. Рассмотрим сначала случай, когда $\operatorname{dim} C<\infty$. Пусть $i$ обозначает отображение включения $\varphi(C) \rightarrow B$. Сюръективный морфизм коалгебр $\varphi: C \rightarrow \varphi(C)$ индуцирует инъективный морфизм алгебр

$$
\varphi(C)^{*} \otimes B \cong \operatorname{Hom}(\varphi(C), B) \hookrightarrow \operatorname{Hom}(C, B) \cong C^{*} \otimes B,
$$

при котором $i \mapsto \varphi$, так что образ $i$ в $C^{*} \otimes B$ обратим. В силу леммы 2.1 получаем $i^{-1} \in \varphi(C)^{*} \otimes B$.

Пусть $C$ - произвольная коалгебра. Если $C^{\prime}-$ конечномерная подкоалгебра в $C$, то подкоалгебра $\varphi\left(C^{\prime}\right)$ слабо обратима в $B$ ввиду уже рассмотренного случая, поскольку $\left.\varphi\right|_{C^{\prime}}$ обратимо в $\operatorname{Hom}\left(C^{\prime}, B\right)$. Как известно, $C$ есть сумма семейства $\mathscr{F}$ своих конечномерных подкоалгебр [4; теорема 2.2.1]. Теперь заключение леммы вытекает из следствия 1.2, поскольку $\varphi(C)=\sum_{C^{\prime} \in \mathscr{F}} \varphi\left(C^{\prime}\right)$.

Теорема 2.3. Слабо конечная биалгебра $B$ имеет наибольшую подалгебру Хопфа $\mathscr{H}(B)$, а такэе наибольшую подалгебру Хопфа с биективным антиподом $\mathbb{H}(B)$. При этом $\mathscr{H}(B)$ (соответственно $\mathbb{H}(B))$ совпадает с наибольшей обратимой в $B$ (соответственно в $B$ и в $B^{\text {op }}$ ) подкоалгеброй. Для произвольной алгебры Хопфа (соответственно алгебры Хопфа с биективным антиподом) $H$ морфизмы биалгебр $H \rightarrow B$ находятся в биективном соответствии с морфизмами алгебр Хопфа $H \rightarrow \mathscr{H}(B)$ (соответственно $H \rightarrow \mathbb{H}(B))$.

Доказательство. Обозначим через $C$ наибольшую обратимую в $B$ подкоалгебру. Наделим $C \otimes C$ структурой тензорного произведения коалгебр [4; раздел 3.0]. Отображение $\varphi: C \otimes C \rightarrow B$, задаваемое умножением в $B$, является морфизмом коалгебр. Кроме того, $\varphi$ имеет обратный

$$
\psi: C \otimes C \rightarrow B, \quad \psi(a \otimes b)=s_{C}(b) s_{C}(a),
$$


в алгебре $\operatorname{Hom}(C \otimes C, B)$. Подкоалгебра $C^{2}=C C=\varphi(C \otimes C)$ слабо обратима в $B$ по лемме 2.2. В силу функториальности $s_{C^{2}} \circ \varphi=\psi$, т.е.

$$
s_{C^{2}}(a b)=s_{C}(b) s_{C}(a) \in C^{2} \quad \text { для } \quad a, b \in C .
$$

Значит, подкоалгебра $C^{2}$ обратима в $B$, что дает $C^{2} \subseteq C$. Так как $k 1_{B}$ - обратимая в $B$ подкоалгебра, то $1_{B} \in C$. Таким образом, $C$ - подбиалгебра в $B$. Поскольку $s_{C}: C \rightarrow C$ является обратным для тождественного отображения $\operatorname{id}_{C}$ в алгебре $\operatorname{Hom}(C, C)$, то $C$ подалгебра Хопфа. Любая подалгебра Хопфа в $B$ является обратимой в $B$ подкоалгеброй и, следовательно, содержится в $C$.

Пусть $f: H \rightarrow B$ - морфизм биалгебр, где $H$ - алгебра Хопфа с антиподом $s_{H}: H \rightarrow H$. Тогда $f^{-1}=f \circ s_{H}$ в алгебре $\operatorname{Hom}(H, B)$. По лемме $2.2 f(H)$ - слабо обратимая подкоалгебра в $B$, так что $s_{f(H)}: f(H) \rightarrow B$ определено. В силу функториальности $f^{-1}=s_{f(H)} \circ f$ в $\operatorname{Hom}(H, B)$. Отсюда

$$
\operatorname{Im} s_{f(H)}=\operatorname{Im}\left(s_{f(H)} \circ f\right)=\operatorname{Im}\left(f \circ s_{H}\right) \subseteq f(H),
$$

т.е. $s_{f(H)}$ дает антипод биалгебры $f(H)$. Следовательно, $f(H)$ - подалгебра Хопфа и, в частности, $f(H) \subseteq C$. Равенство $f \circ s_{H}=s_{f(H)} \circ f$ означает, что $f$ - морфизм алгебр Хопфа $H \rightarrow f(H)$ [4; лемма 4.0.4].

Рассмотрим теперь наибольшую подкоалгебру $D \subseteq B$, обратимую в $B$ и в $B^{\text {ор }}$. Как и выше, проверяется, что подкоалгебра $D^{2}$ обратима в $B$ и в $B^{\text {op }}$. Это влечет, что $D$ - подалгебра Хопфа в $B$ и в $B^{\text {op }}$. Для алгебры Хопфа $H$ условие, что биалгебра $H^{\text {op }}$ - алгебра Хопфа, равносильно биективности антипода $H$ [8; предложение 1.6.2]. В частности, $D$ имеет биективный антипод. Если $f: H \rightarrow B$ - морфизм биалгебр, где $H$ - алгебра Хопфа с биективным антиподом, то $f(H)$ является подалгеброй Хопфа в $B$ и в $B^{\text {ор }}$, что влечет $f(H) \subseteq D$.

СледСтвиЕ 2.4. Если $J$ - биидеал алгебры Хопфа $H$, причем биалгебра $H / J$ слабо конечна, то $J$ является идеалом Хопфа. В частности, любой биидеал является идеалом Хопфа в случае, когда $Н$ нётерова справа или слева, а также в случае, когда $Н$ удовлетворяет полиномиальному тождеству.

ДоказАтельство. Пусть $B=H / J$ и $\pi: H \rightarrow B$ - каноническая проекция. Так как $B$ слабо конечна, то $B=\pi(H) \subseteq \mathscr{H}(B)$. Следовательно, $B$ - алгебра Хопфа, а $J$ идеал Хопфа. Свойства кольца быть нётеровым с одной из сторон или удовлетворять полиномиальному тождеству наследуется всеми факторкольцами, которые в этом случае будут слабо конечными. Отсюда вытекает второе утверждение.

В [3; теорема 1] утверждение о том, что $J$ является идеалом Хопфа, сформулировано для нескольких случаев: $H / J$ конечномерна, $H / J$ коммутативна, $H$ точечна, $H$ кокоммутативна. То же самое заключение верно в предположении о том, что $H$ - строго коплоский $H / J$-комодуль справа или слева [9; следствие 1.5$]$.

В [3] приведен также пример биидеала некоторой алгебры Хопфа, не являющегося идеалом Хопфа. Мы предполагаем, что существуют две алгебры Хопфа $H_{1}, H_{2}$ и биидеал $J$ алгебры Хопфа $H_{1} \otimes H_{2}$, который не является идеалом Хопфа и имеет нулевые пересечения с $H_{1} \otimes 1$ и с $1 \otimes H_{2}$. В этом случае $H_{1}, H_{2}$ будут вкладываться в биалгебру $B=\left(H_{1} \otimes H_{2}\right) / J$ и порождать всю $B$, так что $B$ не будет содержать наибольшей подалгебры Хопфа. В качестве $H_{1}, H_{2}$ придется взять весьма большие алгебры Хопфа например, свободные алгебры Хопфа, построенные в [10], - и проверка высказанного утверждения потребует громоздких выкладок, связанных с построением базиса Гребнеpa. 


\section{СПИСОК ЦИТИРОВАННОЙ ЛИТЕРАТУРЫ}

[1] S. Montgomery, Comm. Algebra, 11:6 (1983), 595-610. [2] L. H. Rowen, Ring Theory, Vol. I, Pure Appl. Math., 127, Acad. Press, Boston, MA, 1988. [3] W. D. Nichols, Comm. Algebra, 6:17 (1978), 1789-1800. [4] M. E. Sweedler, Hopf Algebras, Math. Lecture Note Ser., W. A. Benjamin, New York, 1969. [5] S. Montgomery, Hopf Algebras and Their Actions on Rings, CBMS Regional Conf. Ser. in Math., 82, Amer. Math. Soc., Providence, RI, 1993. [6] T.Y. Lam, Exercises in Classical Ring Theory, Problem Books in Math., Springer-Verlag, New York, NY, 2003. [7] K. R. Goodearl, Comm. Algebra, 15:3 (1987), 589-609. [8] L. A. Lambe, D. E. Radford, Introduction to the Quantum Yang-Baxter Equation and Quantum Groups. An Algebraic Approach, Math. Appl., 423, Kluwer Acad. Publ., Dordrecht, 1997. [9] M. Takeuchi, Comm. Algebra, 22:7 (1994), 2503-2523. [10] M. Takeuchi, J. Math. Soc. Japan, 23 (1971), 561-582.

\section{М. С. Еряшкин}

Казанский государственный университет

С. М. Скрябин

НИИ математики и механики им. Н. Г. Чеботарева, Казанский государственный университет

E-mail: Serge.Skryabin@ksu.ru
Поступило

28.11.2008

Исправленный вариант

23.03.2009 